\title{
44. On Metric General Connections
}

\author{
By Tominosuke ŌTSUKI \\ Tokyo Institute of Technology, Tokyo \\ (Comm. by Z. Suetuna, M.J.A., April 12, 1961)
}

In this note, the author will show that the Levi-Civita's connections of Riemann spaces can be generalized in the theory of general connections under some conditions on an $n$-dimensional differentiable manifold $\mathfrak{X}$. He will use the notations in [3].

1. A tensor $P$ of type $(1,1)$ is called normal when $P$ as a homomorphism of the tangent bundle $T(\mathfrak{X})$ of $\mathfrak{X}$ is an isomorphism on each $P\left(T_{x}(\mathfrak{X})\right)=P_{x}(\mathfrak{X}), x \in \mathfrak{X}$, and $\operatorname{dim} P_{x}(\mathfrak{X})$ is constant. Let us assume that $P$ is normal and put $\operatorname{dim} P_{x}(\mathfrak{X})=m$. If we put $N_{x}(\mathfrak{X})=$ the kernel of $P$ on $T_{x}(\mathfrak{X})$, then we have

$$
T_{x}(\mathfrak{X})=P_{x}(\mathfrak{X})+N_{x}(\mathfrak{X}) .
$$

According to the direct sum decomposition of $T(\mathfrak{X})$, we define two projections $A$ and $N$ which map $T_{x}(\mathfrak{X})$ onto $P_{x}(\mathfrak{X})$ and $N_{x}(\mathfrak{X})$ respectively at each point $x$ of $\mathfrak{X}$. $A$ and $N$ may be considered as tensors of type $(1,1)$ of $\mathfrak{X}$. Clearly we have $A+N=I, A^{2}=A, N^{2}=N, A N=N A=0$, $A P=P A=P$ and $N P=P N=0$, where $I$ denotes the fundamental unit tensor of type $(1,1)$.

Now, we say that a normal tensor $P$ is orthogonally related with a non-singular symmetric tensor $G=g_{i j} d u^{i} \otimes d u^{j}$, if $P_{x}(\mathfrak{X})$ and $N_{x}(\mathfrak{X})$ are mutually orthogonal with respect to $G$, regarding $G$ as a metric tensor.

A general connection $\Gamma$, which is locally written as

$$
\Gamma=\partial u_{i} \otimes\left(P_{j}^{i} d^{2} u^{j}+\Gamma_{j h}^{i} d u^{j} \otimes d u^{h}\right),{ }^{1)} \partial u_{i}=\partial / \partial u^{i},
$$

is called normal, if the tensor $P=\lambda(\Gamma)^{2)}=\partial u_{i} \otimes P_{j}^{i} d u^{j}$ is normal.

A normal general connection $\Gamma$ is called proper, ${ }^{8)}$ if the tensor of type $(1,2)$ with local components $N_{k}^{i} \Gamma_{j^{i}}^{k}$ vanishes, where $N_{j}^{i}$ are the local components of the tensor $N$.

We say that a general connection $\Gamma$ satisfies the metric condition for a symmetric covariant tensor $G=g_{i j} d u^{i} \otimes d u^{j}$, if

$$
D G=g_{i j, h} d u^{i} \otimes d u^{j} \otimes d u^{h}=0,
$$

where $D G$ denotes the covariant differential of $G$ with respect to $\Gamma .{ }^{4)}$ On the metric condition, the following theorem holds good as in the

1) See [3].

2) See [3], §2.

3) On the geometrical meaning of this condition, see Theorem 5.2 of [4]. In general, $\Gamma_{j h}^{i}$ are not local components of a tensor of type $(1,3)$ as the classical affine connections but $N_{k}^{i} \Gamma_{\mathrm{g} h}^{k}$ are so.

4) See (2.15) of [3]. 
classical case.

Theorem 1. Let $\Gamma$ be a metric general connection with respect to a symmetric covariant tensor $G$ of order 2. For any two contravariant vectors with local components $v^{i}$ and $w^{i}$ defined on a curve $u^{i}=u^{i}(t)$ along which they are covariantly constant, the scalar $g_{h k} P_{i}^{h} P_{j}^{k} v^{i} w^{j}$ is constant..$^{5)}$ Conversely, if $\Gamma$ has the property for any curve, then $\Gamma$ is metric with respect to $G$.

Proof. The metric condition (1) is written as

$$
g_{i j, h}=\frac{\partial g_{l k}}{\partial u^{h}} P_{i}^{l} P_{j}^{k}-g_{l k} \Lambda_{i h}^{l} P_{j}^{k}-g_{l k} P_{i}^{l} \Lambda_{j h}^{k}=0,
$$

where

$$
\Lambda_{j h}^{i}=\Gamma_{j h}^{i}-\frac{\partial P_{j}^{i}}{\partial u^{h}}
$$

Clearly, $g_{i j, h}$ can be also written as

$$
g_{i j, h}=\frac{\partial}{\partial u^{h}}\left(g_{l k} P_{i}^{l} P_{j}^{k}\right)-g_{l k} \Gamma_{i h}^{l} P_{j}^{k}-g_{l k} P_{i}^{l} \Gamma_{j h \text {. }}^{k}
$$

Now, let $V=v^{i} \partial u_{i}, W=w^{i} \partial u_{i}$ be covariantly constant along a curve $u^{i}=u^{i}(t)$, then it must hold good

$$
D v^{i}=P_{j}^{i} d v^{j}+\Gamma_{j h}^{i} v^{j} d u^{h}=0, \quad D w^{i}=P_{j}^{i} d w^{j}+\Gamma_{j h}^{i} w^{j} d u^{h}=0
$$

along the curve. Hence, we have

$$
\begin{aligned}
d\left(g_{l k} P_{i}^{l} P_{j}^{k} v^{i} w^{j}\right) & =d\left(g_{l k} P_{i}^{l} P_{j}^{k}\right) v^{i} w^{j}+g_{l k}\left(P_{i}^{l} d v^{i}\right) P_{j}^{k} w^{j}+g_{l k} P_{i}^{l} v^{i}\left(P_{j}^{k} d w^{j}\right) \\
& =\left[d\left(g_{l k} P_{i}^{l} P_{j}^{k}\right)-g_{l k} \Gamma_{i h}^{l} P_{j}^{k} d u^{h}-g_{l k} P_{i}^{l} \Gamma_{j k}^{k} d u^{h}\right] v^{i} w^{j} \\
& =g_{i j, h} v^{i} w^{j} d u^{h} .
\end{aligned}
$$

Since, at any point of a curve, we have solutions of (3) with any initial values at the point, the condition: $g_{i j, h}=0$ is equivalent to the condition: $d\left(g_{l k} P_{i}^{l} P_{j}^{k} v^{i} w^{j}\right)=0$ for any curve and any two contravariant vector fields $v^{i}$ and $w^{i}$ covariantly constant along the curve. q.e.d.

2. Now we shall prove the following

Theorem 2. Let $P=P_{j}^{i} \partial u_{i} \otimes d u^{j}$ and $G=g_{i j} d u^{i} \otimes d u^{j}$ be a normal tensor and a non-singular symmetric tensor on $¥$ such that $P$ is orthogonally related with $G$. Then, there exist normal general connections $\Gamma$ which satisfy the following conditions:
(i) $P=\lambda(\Gamma)$,
(ii) $\Gamma$ is proper, and
(iii) $\Gamma$ is metric with respect to $G$.

Furthermore, if we add the condition:

$$
S_{k h}^{i} A_{j}^{k}=\frac{1}{2} A_{i}^{i}\left(P_{k ; h}^{l}-P_{h ; k}^{l}\right) A_{j}^{k},
$$

where $A_{j}^{i}$ are the local components of $A, S_{j h}^{i}=\frac{1}{2}\left(\Gamma_{j h}^{i}-\Gamma_{h j}^{i}\right)$

and the semi-colon ";" denotes the covariant derivatives with respect to the Levi-Civita's connection made by $G$, then $\Gamma$ is uniquely determined.

5) For general connections, the covariant differentiation and the contraction are not necessarily commutative. See [3], \$2. 
The condition (iv) is a generalization of the symmetric condition in the classical case, because we have $A_{j}^{i}=\delta_{j}^{i}$ and $P_{j ; h}^{i}=0$, when $P_{j}^{i}=\delta_{j}^{i}$.

Proof. Now, let be given two tensors $P$ and $G$ as stated in the theorem and assume that there exists a normal general connection $\Gamma$ satisfying the conditions (i), (ii) and (iii).

If we put

$$
\bar{g}_{i j}=g_{k h} P_{i}^{k} P_{j}^{n}, \bar{\Gamma}_{j h}^{i}=\frac{1}{2}\left(\Gamma_{j h}^{i}+\Gamma_{h j}^{i}\right), S_{j_{h}}^{i}=\frac{1}{2}\left(\Gamma_{j h}^{i}-\Gamma_{h j}^{i}\right),
$$

the condition (iii) can be written as

$$
\frac{\partial \bar{g}_{i j}}{\partial u^{h}}=\bar{\Gamma}_{i k h} P_{j}^{k}+\bar{\Gamma}_{j k h} P_{i}^{k}+S_{i k h} P_{j}^{k}+S_{j k h} P_{i}^{k},
$$

where

$$
\bar{\Gamma}_{i k h}=g_{k l} \bar{\Gamma}_{i h}^{l}, \quad S_{i k h}=g_{k l} S_{i h}^{l} .
$$

As easily seen, $S_{j h}^{i}$ are the local components of a tensor of type $(1,2)$ as in the classical case. If we denote the Christoffel symbols of the first kind made by $\bar{g}_{i j}$ by

$$
\left[\overline{i j, h]}=\frac{1}{2}\left(\frac{\partial \bar{g}_{i h}}{\partial u^{j}}+\frac{\partial \bar{g}_{h j}}{\partial u^{i}}-\frac{\partial \bar{g}_{i j}}{\partial u^{h}}\right),\right.
$$

then (4) is clearly equivalent to

$$
[\overline{i j, h}]=\bar{\Gamma}_{i k j} P_{h}^{k}+S_{h k i} P_{j}^{k}+S_{h k j} P_{i}^{k} .
$$

Now, let $Q$ be the homomorphism of $T(\mathfrak{X})$ which operates as $Q=P^{-1}$ on each $P_{x}(\mathfrak{X})$ and $Q=0$ on each $N_{x}(\mathfrak{X})$. Then we have easily $P Q=Q P=A, Q N=N Q=0$.

Let $Q_{j}^{i}$ be the local components of $Q$. Then, we get from (5)

$$
[\overline{i j, l}] Q_{h}^{z}=\bar{\Gamma}_{i l j} A_{h}^{l}+\left(S_{l k i} P_{j}^{k}+S_{l k j} P_{i}^{k}\right) Q_{h}^{l}
$$

and

$$
[\overline{i j, l}] N_{h}^{i}=\left(S_{l k i} P_{j}^{k}+S_{l k j} P_{i}^{k}\right) N_{h}^{l} .
$$

Making use of the relations between $A, N, P$ and $Q$, we can easily see that (5) is derived from (6) and (7).

The condition (ii) can be written as

and

(9)

$$
N_{k}^{i} \bar{\Gamma}_{j h}^{k}=0
$$

$$
N_{k}^{i} S_{j h}^{k}=0 \text {. }
$$

Therefore, in order to obtain a normal general connection $\Gamma$ satisfying the conditions (i), (ii) and (iii), it is sufficient that we solve firstly the equations (7) and (9) with respect to $S_{j h}^{i}=-S_{h_{j}}^{i}$, and secondly the equations (6) and (8) with respect to $\bar{\Gamma}_{j h}^{i}=\bar{\Gamma}_{h j}^{i}$, using the solution $S_{j h}^{i}$ of (7) and (9).

In the first place, we shall show that there exists a solution of (7) and (9) under the condition between $P$ and $G$. We have

$$
2[\overline{i j, l}] N_{h}^{i}=\left\{\frac{\partial}{\partial u^{j}}\left(g_{s t} P_{i}^{s} P_{l}^{t}\right)+\frac{\partial}{\partial u^{i}}\left(g_{s t} P_{j}^{s} P_{l}^{t}\right)-\frac{\partial}{\partial u^{l}}\left(g_{s t} P_{i}^{s} P_{j}^{t}\right)\right\} N_{h}^{l}
$$


that is

$$
\begin{aligned}
= & \left\{g_{k t}\left(\frac{\partial P_{l}^{t}}{\partial u^{i}}-\frac{\partial P_{i}^{t}}{\partial u^{l}}\right) P_{j}^{k}+g_{k t}\left(\frac{\partial P_{l}^{t}}{\partial u^{j}}-\frac{\partial P_{j}^{t}}{\partial u^{l}}\right) P_{i}^{k}\right. \\
& \left.-([s l, t]+[t l, s]) P_{i}^{s} P_{j}^{t}\right\} N_{h}^{l},
\end{aligned}
$$

$$
[\overline{i j, l}] N_{h}^{l}=\left\{\frac{1}{2} g_{k t}\left(P_{l: i}^{t}-P_{i ; l}^{t}\right) P_{j}^{k}+\frac{1}{2} g_{k t}\left(P_{l ; j}^{t}-P_{j ; l}^{t}\right) P_{i}^{k}\right\} N_{h}^{l},
$$

where $[i j, h]$ are the Christoffel symbols of the first kind made by $g_{i j}$. Comparing (10) with (7), we define a tensor of type $(1,3)$ with local components

$$
\bar{S}_{j h}^{i}=\frac{1}{2} A_{k}^{i}\left(P_{j ; h}^{k}-P_{h ; j}^{k}\right)
$$

then we have

$$
\begin{aligned}
& \left(\bar{S}_{l k i} P_{j}^{k}+\bar{S}_{l k j} P_{i}^{k}\right) N_{h}^{l}=g_{k t}\left(\bar{S}_{l i}^{t} P_{j}^{k}+\bar{S}_{l j}^{t} P_{i}^{k}\right) N_{h}^{l} \\
= & \left\{\frac{1}{2} g_{k t}\left(P_{l ; i}^{t}-P_{i ; l}^{t}\right) P_{j}^{k}+\frac{1}{2} g_{k t}\left(P_{l ; j}^{t}-P_{j ; l}^{t}\right) P_{i}^{k}\right\} N_{h}^{l} \\
- & \frac{1}{2}\left\{g_{k s} N_{t}^{s} P_{j}^{k}\left(P_{l ; i}^{t}-P_{i ; l}^{t}\right)+g_{k s} N_{t}^{s} P_{i}^{k}\left(P_{l ; j}^{t}-P_{j ; l}^{t}\right)\right\} N_{h}^{l} \\
= & {[\overline{i j, l]}] N_{h}^{l}, }
\end{aligned}
$$

since we have $g_{i j} P_{k}^{i} N_{h}^{j}=0$. On the other hand, we have

$$
N_{k}^{i} \bar{S}_{j h}^{k}=\frac{1}{2} N_{k}^{i} A_{l}^{k}\left(P_{j ; h}^{l}-P_{h ; j}^{l}\right)=0 \text {. }
$$

Thus, we have proved that the tensor $\bar{S}_{j h}^{i}$ is a solution of (7) and (9).

Now, if we put

$$
S_{j h}^{i}-\bar{S}_{j h}^{i}=X_{j h}^{i}=-X_{h j}^{i},
$$

they must satisfy the equations

$$
\begin{gathered}
\left(X_{l k i} P_{j}^{k}+X_{l k j} P_{i}^{k}\right) N_{h}^{l}=0, \\
N_{k}^{i} X_{j h}^{k}=0 .
\end{gathered}
$$

Furthermore, supposing the condition (iv), it can be written as

$$
X_{k h}^{i} A_{j}^{k}=0 \text {. }
$$

It is equivalent to

$$
X_{k h}^{i} N_{j}^{k}=X_{j h}^{i}
$$

and so (12) can be written as

$$
X_{h k i} P_{j}^{k}+X_{h k j} P_{i}^{k}=0 \text {. }
$$

Hence $Y_{i h j}=X_{i k j} P_{h}^{k}$ are skew-symmetric with respect to the indices $i$, $h, j$. Using (14), we get

hence

$$
\begin{aligned}
Y_{i k j} A_{h}^{k} & =X_{i l j} P_{k}^{l} A_{h}^{k}=X_{i l j} P_{h}^{l}=Y_{i h j} \\
& =-Y_{k i j} A_{h}^{k}=-X_{k l j} A_{h}^{k} P_{i}^{l}=0,
\end{aligned}
$$

$$
X_{i k j} A_{h}^{k}=0 \text {. }
$$

On the other hand, from the assumption that $P$ is orthogonally related with $G$, we have

$$
g_{i j} A_{k}^{s} N_{h}^{j}=0
$$


Using these relations (15), (13) and (16), we have

$$
X_{i h j}=X_{i k j} N_{h}^{k}=X_{i j}^{l} g_{k l} N_{h}^{k}=X_{i j}^{t} g_{k l} A_{t}^{l} N_{h}^{k}=0 .
$$

Thus, we have proved that under the conditions (i)-(iv), there exists a unique solution $S_{j h}^{i}$ which is the skew-symmetric part of $\Gamma_{j h}^{i}$.

In the next place, we shall show that there exists a unique solution $\bar{\Gamma}_{j h}^{i}$ of (6) and (8) under the conditions (i), (ii), and (iii) regarding $S_{j h}^{i}$ as a known tensor.

Let us take a local field of frame $\left\{V_{\lambda}\right\}$ of the tangent bundle $T(\mathfrak{X})$ of $\mathfrak{X}$ such that $\left\{V_{1}, \cdots, V_{m}\right\}$ and $\left\{V_{m+1}, \cdots, V_{n}\right\}$ are frames of $P_{x}(\mathfrak{X})$ and $N_{x}(\mathfrak{X})$ at each point $x$ respectively. Let $\left\{U^{\lambda}\right\}$ be the dual frame of $\left\{V_{\lambda}\right\}$. Then we have $\left.A_{j}^{i}=V_{\alpha}^{i} U_{j}^{\alpha}, N_{j}^{i}=V_{B}^{i} U_{j}^{B} .{ }^{6}\right) \quad U^{\lambda i}=g^{i j} U_{j}^{\lambda}$ can be written as $U^{i t}=C^{\lambda \mu} V_{\mu}^{i}$, hence we have

$$
C^{\lambda \mu}=g^{i j} U_{i}^{\lambda} U_{j}^{\mu}=C^{\mu \lambda},\left|C^{\lambda \mu}\right| \neq 0 .
$$

If we put

$$
C_{\lambda \mu}=g_{i j} V_{\lambda}^{i} V_{\mu}^{j}=C_{\mu \lambda},
$$

the matrix $\left(C_{\lambda \mu}\right)$ is the inverse of the matrix $\left(C^{2 \mu}\right)$. By virtue of the assumption of this theorem, we have

$$
C_{\alpha A}=C^{\beta B}=0 .
$$

Now, (8) is clearly equivalent to

and so we have

$$
U_{k}^{A} \bar{\Gamma}_{i j}^{k}=0, \quad A=m+1, \cdots, n,
$$

hence

$$
\bar{\Gamma}_{i l j} U^{A l}=\bar{\Gamma}_{i l j} C^{A B} V_{B}^{\iota}=0,
$$

$$
\bar{\Gamma}_{i l j} N_{h}^{l}=0 .
$$

From (6) and (17), we get

and

$$
\bar{\Gamma}_{i h j}=\left([\overline{i j, l}]-S_{l k i} P_{j}^{k}-S_{l k j} P_{i}^{k}\right) Q_{h}^{l}
$$

$$
\bar{\Gamma}_{i j}^{h}=\left([\overline{i j, l}]-S_{l k i} P_{j}^{k}-S_{l k j} P_{i}^{k}\right) Q_{p}^{l} g^{p h} .
$$

Conversely, $\bar{\Gamma}_{i_{j}}^{h}$ given by (18) satisfy (6) and (8), as is easily seen.

Lastly, we must show that $\partial u_{i} \otimes\left(P_{j}^{i} d^{2} u^{j}+\left(\overline{\Gamma_{j h}^{i}}+S_{j h}^{i}\right) d u^{j} \otimes d u^{h}\right)$ is a general connection. It is sufficient to show that $\partial u_{i} \otimes\left(P_{j}^{i} d^{2} u^{j}+\bar{\Gamma}_{j h}^{i} d u^{j}\right.$ $\left.\otimes d u^{n}\right)$ is a general connection. Here, let us denote the components in another coordinate system $v^{2}$ by the notations with stars. Then we have

$$
\begin{aligned}
\bar{\Gamma}_{i j}^{h} & =\left(\bar{g}_{\lambda \mu}^{*} \frac{\partial^{2} v^{\lambda}}{\partial u^{j} \partial u^{i}} \frac{\partial v^{\mu}}{\partial u^{l}}+\left([\overline{\lambda \mu, \rho}]^{*}-S_{\rho \tau \lambda}^{*} P_{\mu}^{* \tau}-S_{\rho \tau \mu}^{*} P_{\lambda}^{* \tau}\right) \frac{\partial v^{\lambda}}{\partial u^{i}} \frac{\partial v^{\mu}}{\partial u^{j}} \frac{\partial v^{\rho}}{\partial u^{l}}\right) Q_{k}^{l} g^{k h} \\
& =\frac{\partial u^{h}}{\partial v^{\nu}}\left(g^{* \nu \rho} Q_{\rho}^{* \mu} \bar{g}_{\mu \lambda}^{*} \frac{\partial^{2} v^{\lambda}}{\partial u^{j} \partial u^{i}}+\bar{\Gamma}_{\lambda \mu}^{* \nu} \frac{\partial v^{2}}{\partial u^{i}} \frac{\partial v^{\mu}}{\partial u^{j}}\right) .
\end{aligned}
$$

6) Indices run as follows: $\lambda, \mu, \nu, \cdots=1,2, \cdots, n ; \alpha, \beta, \cdots=1,2, \cdots, m ; A, B, \cdots$ $=m+1, \cdots, n$. 
Since we have

the above equation can be written as

$$
\begin{aligned}
g^{i k} Q_{k}^{h} \bar{g}_{h j} & =g^{i k} Q_{k}^{h} g_{l t} P_{h}^{l} P_{j}^{t}=g^{i k} A_{k}^{l} g_{l h} P_{j}^{h} \\
& =P_{j}^{i}-g^{i k} g_{l h} N_{k}^{l} P_{j}^{h}=P_{j}^{i},
\end{aligned}
$$

$$
\bar{\Gamma}_{i j}^{h}=\frac{\partial u^{h}}{\partial v^{\nu}}\left(P_{\lambda{ }^{2}}^{*_{\nu}} \frac{\partial^{2} v^{\lambda}}{\partial u^{j} \partial u^{i}}+\bar{\Gamma}_{\lambda \mu}^{* \nu} \frac{\partial v^{\lambda}}{\partial u^{i}} \frac{\partial v^{\mu}}{\partial u^{j}}\right)
$$

This shows that $\partial u_{i} \otimes\left(P_{j}^{i} d^{2} u^{j}+\overline{\Gamma_{j h}^{i}} d u^{j} \otimes d u^{h}\right)$ determines a general connection. Thus, we have proved the theorem.

q.e.d.

\section{References}

[1] T. Ötsuki: On tangent bundles of order 2 and affine connections, Proc. Japan Acad., 34, 325-330 (1958).

[2] - : Tangent bundles of order 2 and general connections, Math. J. Okayama Univ., 8, 143-179 (1958).

[3] - : On general connections I, Math. J. Okayama Univ., 9, 99-164 (1960).

[4] - : On general connections II, Math. J. Okayama Univ., 10, 113-124 (1961). 